\title{
Development of an ASIC for Si/CdTe detectors in a radioactive substance visualizing system
}

\author{
Atsushi Harayama ${ }^{a}$, Shin'ichiro Takeda ${ }^{a}$, Goro Sato ${ }^{\mathrm{b}, \mathrm{a}}$, Hirokazu Ikeda ${ }^{\mathrm{a}}$, \\ Shin Watanabe ${ }^{\mathrm{a}}$ and Tadayuki Takahashi ${ }^{\mathrm{a}}$ \\ ${ }^{a}$ Institute of Space and Astronautical Science, Japan Aerospace Exploration Agency, \\ Sagamihara, Kanagawa 229-8510, Japan \\ ${ }^{b}$ RIKEN Nishina Center, 2-1 Hirosawa, Wako, Saitama 351-0198, Japan
}

\section{Abstract}

We report on the recent development of a 64-channel analog front-end ASIC for a new gamma-ray imaging system designed to visualize radioactive substances. The imaging system employs a novel Compton camera which consists of silicon $(\mathrm{Si})$ and cadmium telluride $(\mathrm{CdTe})$ detectors. The ASIC is intended for the readout of pixel/pad detectors utilizing $\mathrm{Si} / \mathrm{CdTe}$ as detector materials, and covers a dynamic range up to $1.4 \mathrm{MeV}$. The readout chip consists of 64 identical signal channels and was implemented with X-FAB $0.35 \mu \mathrm{m}$ CMOS technology. Each channel contains a charge-sensitive amplifier, pole-zero cancellation circuit, low-pass filter, comparator, and a samplehold circuit, along with a Wilkinson-type A-to-D converter. We observed an equivalent noise charge of $\sim 500 \mathrm{e}^{-}$and noise slope of $\sim 5 \mathrm{e}^{-} / \mathrm{pF}$ (r.m.s.) with power consumption of $2.1 \mathrm{~mW}$ per channel. The chip works well when connected to Schottky CdTe diodes, and delivers spectra with good energy resolution, such as $\sim 12 \mathrm{keV}$ (FWHM) at $662 \mathrm{keV}$ and $\sim 24 \mathrm{keV}(\mathrm{FWHM})$ at $1.33 \mathrm{MeV}$.

Keywords: ASIC; Gamma ray; Si/CdTe; Analog front-end 


\section{Introduction}

A new gamma-ray imaging system that can visualize radioactive substances is urgently needed to help decontamination efforts in Fukushima. The imaging system employs a Compton camera that offers many advantages over traditional gamma cameras. Because it directly locates the source by Compton reconstruction, this system needs no mechanical collimator or heavy high- $\mathrm{Z}$ material (e.g. $\mathrm{Pb}$ ) to cover the imaging detector in order to avoid contamination by background gamma rays.

We have developed a novel Compton camera which consists of silicon ( $\mathrm{Si}$ ) and cadmium telluride ( $\mathrm{CdTe}$ ) semiconductor detectors for the ASTRO-H satellite mission scheduled for launch in 2015[1]. The original technologies of low-noise silicon devices[2], high resolution Schottky CdTe diodes[3], and a dedicated low-noise analog ASIC allow the tracking of low energy gamma rays, thereby realizing an energy coverage of $100 \mathrm{keV}$ to a few MeV[4]. By employing these technologies, we have fabricated a dedicated prototype for decontamination within the evacuation zone in Fukushima[6]. The prototype consists of two layers of Si double-sided strip detectors and three layers of CdTe double-sided strip detectors, with a detector area of $3.2 \times 3.2 \mathrm{~cm}$ and a strip pitch of $250 \mu \mathrm{m}$.

The system's imaging performance was reported as $3.8^{\circ}$ (FWHM) at 662 $\mathrm{keV}$ for the angular resolution, 2.2\% (FWHM) at $662 \mathrm{keV}$ energy resolution, and almost $2 \pi$ steradian $\left(180^{\circ} \times 180^{\circ}\right)$ for the FoV [5]. Hotspots corresponding to air radiation levels of a few $\mu \mathrm{Sv} / \mathrm{h}$ in the evacuation zone were clearly visible with an exposure of around 40-60 $\min [6]$. 


\section{Circuit Description}

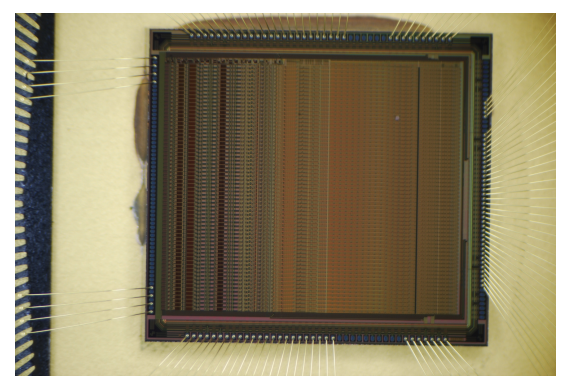

Figure 1: A photograph of the 64-channel front-end ASIC (KW04D64) mounted in a QFP ceramic package. 
$\mu \mathrm{m}$ CMOS technology with a chip size of $7.8 \times 7.1 \mathrm{~mm}^{2}$. Figure 1 shows a photograph of the chip containing 64 readout channels. Each channel contains a charge-sensitive amplifier, pole-zero cancellation circuit, low-pass filter, comparator, and a sample-hold circuit, along with a Wilkinson-type analog-to-digital converter. The bonding pads for signal inputs are located on the left side. The strip pitch is $91.2 \mu \mathrm{m}$ and the pad area is $65 \times 90 \mu \mathrm{m}^{2}$.

Figure 2 shows a signal processing chain for each channel. The detector electrode is DC-coupled to an input of the charge-sensitive amplifier (CSA), while test pulses can be injected via an $\mathrm{AC}$-coupling capacitor $\left(\mathrm{C}_{\mathrm{in}}\right)$. The CSA is based on a folded cascade configuration and utilizes a PMOS transistor as the input transistor. The gate length and width are 1.2 and $8 \mu \mathrm{m}$, respectively, and the $\mathrm{M}$ value (denoting the number of transistors arranged in parallel) is set as 180 . The feedback capacitor $\left(\mathrm{C}_{\mathrm{f}}\right)$ was selectable between 0.25 and $0.50 \mathrm{pF}$ via a CMOS switch, dedicated for high and low gain modes, respectively. A transfer gate-type FET $\left(\mathrm{R}_{\mathrm{f}}\right)$ was employed for the CSA DC feedback component. The CSA output was fed into two shaping amplifiers: a slow shaper for spectroscopy and a fast shaper for triggering. Each amplifier is composed of a pole-zero cancellation circuit $\left(\mathrm{C}_{\mathrm{dif}}, \mathrm{R}_{\mathrm{pz}}\right)$ and a second order low-pass filter. The parameters are set as:

$$
\begin{gathered}
C_{\mathrm{f}} R_{\mathrm{f}}=C_{\mathrm{dif}} R_{\mathrm{pz}} \quad \text { and } \\
C_{1} R_{1}=4 C_{2} R_{2}, \quad C_{3} R_{3}=4 C_{4} R_{4}
\end{gathered}
$$

${ }_{71}$ The peaking times are selectable between 5 and $10 \mu \mathrm{s}$ for the slow shaper, and between 1 and $2 \mu$ s for the fast shaper. The slow shaper output is fed into 3 the sample-and-hold $(\mathrm{S} / \mathrm{H})$ circuit, while the fast shaper output is fed into 
a comparator with a threshold voltage of VTH1. The fast shaper channel is equipped with an automatic offset compensation circuit (OFFSET).

The Wilkinson-type analog-to-digital converter includes a comparator that refers to a ramp voltage common to all the channels, and strobes digits from a Gray-coded counter when the ramp signal exceeds the voltage of $\mathrm{S} / \mathrm{H}$ circuit. The digitized amplitudes are read out by external electronics, e.g. Lab-VIEW system. The analog-to-digital converter includes a common mode noise (CMN) detection mechanism. The CMN could be defined as the median of the pedestal distribution for each event, which can be acquired while the ramp signal is passing through the base-line level.

There are two sparse readout modes in addition to the entire read out mode: one is an analog trigger mode; the other is a digital trigger mode. In the analog trigger mode, one can read only from those channels whose fast shapers deliver amplitudes exceeding VTH1. In the digital trigger mode, the A-to-D converted S/H data are corrected by the CMN, and the read out condition is met if they exceed a predetermined digital threshold. The digital trigger mode can be utilized to improve trigger quality by suppressing the environmental interference.

\section{Circuit Properties}

To evaluate the circuit properties, the ASIC was placed in a QFP ceramic package. The package was held in a burn-in socket mounted on a test board. An interface with a computer was established using a National Instruments PCI-7833R board containing a reconfigurable FPGA and analog-to-digital converters. Lab-VIEW software tools were used to control the readout se- 


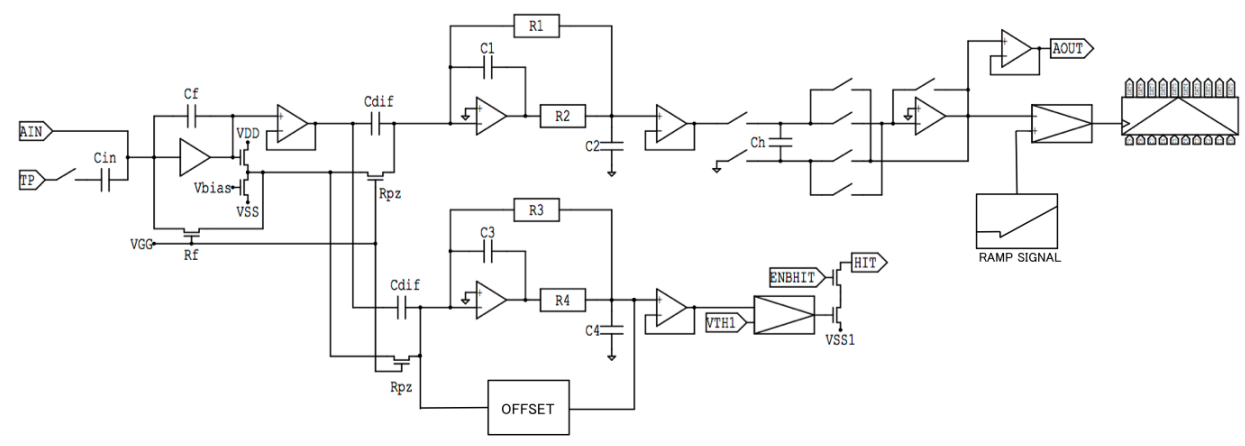

Figure 2: A signal processing chain for each channel.

quence. The interface signals are organized into CMOS inputs. Since CMOS logic gates for the ASIC are operated with $\mathrm{V}_{D D}=+1.65 \mathrm{~V}$ and $\mathrm{V}_{S S}=-$ $1.65 \mathrm{~V}$, internal signals on the chip must be converted from and into LVTTL signals for the PCI-7833R board.

We measured electronic noise by using the outer 8 channels connected to a DIP socket mounted on the test board. Figure 3 shows the equivalent noise charge (ENC) against input capacitance in high gain mode. An equivalent noise charge (ENC) of $500 \mathrm{e}^{-}+5 \mathrm{e}^{-} / \mathrm{pF}$ (r.m.s.) was obtained with power consumption of $2.1 \mathrm{~mW}$ per channel. While the noise slope is consistent with SPICE simulation results, the total noise is significantly larger than the design value. The expected noise level is $237 \mathrm{e}^{-}$for an input capacitance of 0 $\mathrm{pF}$. The large difference in ENC between the simulation and the experimental data is suspected to be a digital-to-analog interference. By inspecting the layout precisely, we found that in some locations analog power lines are unexpectedly narrower and overlap with digital power and signal lines. The exact reason of the excess noise is still under investigation. 
The circuit linearity was also investigated for both high and low gain modes $\left(\mathrm{C}_{\mathrm{f}}=0.25 \mathrm{pF}\right.$ and $\mathrm{C}_{\mathrm{f}}=0.50 \mathrm{pF}$, respectively $)$. Figure 4 shows the results for a typical channel. The output voltage is in the range between 0 to 900 $\mathrm{mV}$, and covers a dynamic range up to $1.4 \mathrm{MeV}$ in high gain mode.

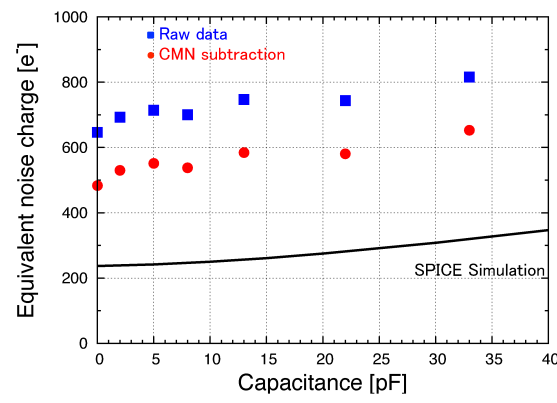

Figure 3: Equivalent noise charge (ENC) against input capacitance in high gain mode. The solid line show results from SPICE simulation. Blue squares indicate the measured raw data; red circles indicate common mode subtracted noise.

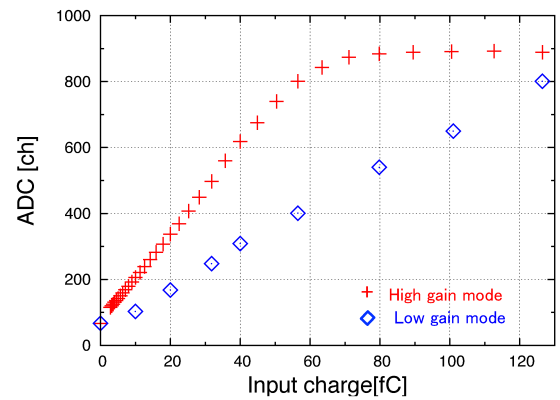

Figure 4: Linearity curves for high and low gain mode. Red crosses indicate high gain mode; Blue squares indicate low gain mode.

\section{Performance test with a Schottky CdTe Diode}

In the performance verification test, we connected the ASIC to a CdTe 


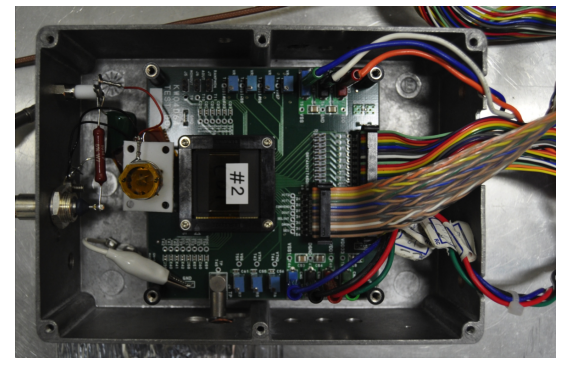

Figure 5: Setup for the spectral measurements with a Schottky CdTe diode detector.

factured by ACRORAD of Japan. With the use of indium (In) and platinum (Pt) for the anode electrode and cathode electrode, respectively, this device works as a Schottky diode. This device has lower leakage current than that of ohmic detectors and allows higher bias voltages to be applied, leading to almost full charge collection[12]. Moreover, the cathode electrode is covered by a guard ring to reduce the detector's leakage current[13]. The CdTe detector is $3.45 \times 3.45 \mathrm{~mm}$ in size and $1 \mathrm{~mm}$ in thickness, and is surrounded by a guard ring $1 \mathrm{~mm}$ in width.

Since spectral performance depends on the leakage current and detector capacitance, the leakage current must be measured before connection to the ASIC. Positive voltages were applied to the indium electrode using a high-voltage sourcemeter(Keithley 2410). As a result, the measured leakage current was $1.5 \mathrm{nA}$ with a bias voltage of $1000 \mathrm{~V}$ at $-15^{\circ} \mathrm{C}$, and the detector capacitance was $2.5 \mathrm{pF}$.

Figure 5 shows the setup for the spectral measurements. An electric wire of about $1 \mathrm{~cm}$ is used for connection between the active area of the cathode electrode and the DIP socket mounted on the test board. The DIP socket is wired to the QFP socket pins corresponding to CSA input. We 


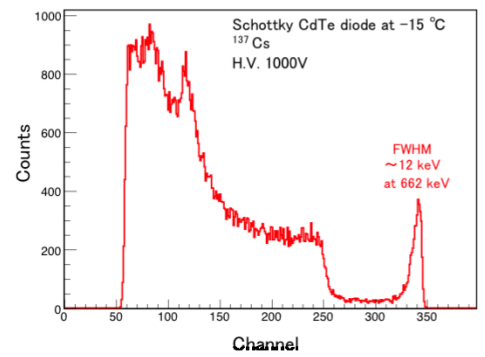

Figure 6: Spectrum of ${ }^{137} \mathrm{Cs}$ with the ASIC connected to a Schottky CdTe diode in high gain mode.

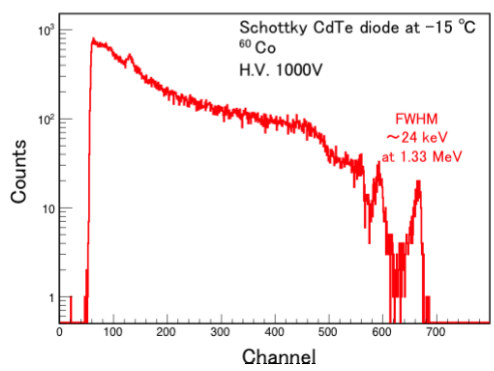

Figure 7: Spectrum of ${ }^{60} \mathrm{Co}$ with the ASIC connected to a Schottky CdTe diode in high gain mode. This is obtained under the same conditions as for the spectrum of ${ }^{137} \mathrm{Cs}$ 


\section{Summary \& Future Plans}

We developed a 64-channel analog front-end ASIC for a new gamma-ray imaging system designed to visualize radioactive substances. We observed an equivalent noise charge of $\sim 500 \mathrm{e}^{-}+5 \mathrm{e}^{-} / \mathrm{pF}$ (r.m.s.) with power consumption of $2.1 \mathrm{~mW}$ per channel. The chip works well when connected to Schottky CdTe diodes, and obtained spectra with good energy resolution, such as $\sim 12$ $\mathrm{keV}$ (FWHM) at $662 \mathrm{keV}$ and $\sim 24 \mathrm{keV}$ (FWHM) at $1.33 \mathrm{MeV}$. The large difference in ENC between the simulation and the experimental data is considered as due to an improper layout of the power lines. An improved version is now being fabricated and will be delivered soon.

\section{Acknowledgments}

This work was supported by the JST-SENTAN program.

\section{References}

[1] T.Takahashi, K. Mitsuda and H. Kunieda, SPIE. vol. 6266, pp. 62660D, 2006

[2] H. Tajima, T.Kamae, S. Uno, T. Nakamoto, Y. Fukazawa, T. Mitani, T. Takahashi, K. Nakazawa, Y. Okada and M. Nomachi, Proc. SPIE, vol. 4851, pp. 875-884, 2003.

[3] T. Takahashi, K. Hirose, C. Matsumoto, K. Takizawa, R. Ohno, T. Ozaki, K. Mori and Y. Tomita, Proc. SPIE. vol. 3446, pp. 29-37, 1998 
[4] S. Takeda, S. Ishikawa, H. Odaka, S. Watanabe, T. Takahashi, K. Nakazawa, H. Tajima, Y. Kuroda, M. Onishi, Y. Fukazawa and H. Yasuda, Proc. SPIE, vol. 6706 pp. 67060S, 2007

[5] S. Takeda, Y. Ichinohe, K. Hagino, H. Odaka, T. Yuasa, S. Ishikawa, T. Fukuyama, S. Saito, T. Sato, G. Sato, S. Watanabe, M. Kokubun, T. Takahashi, M. Yamaguchi, H. Tajima, T. Tanaka, K. Nakazawa, Y. Fukazawa and T. Nakano, Physics Proc., vol. 37, 859- 866, 2012

[6] T. Takahashi, S. Takeda, S. Watanabe and H.Tajima, IEEE NSS/MIC 4199, 2012

[7] H. Ikeda, Nucl. Instr. Meth. A, 569, 98, 2006

[8] T. Kishishita, H. Ikeda, T. Kiyuna, K. Tamura, K. Nakazawa, T. Takahashi, Nucl. Instr. Meth. A 578, 218, 2007

[9] T. Kishishita, H. Ikeda, T. Sakumura, K. Tamura, T. Takahashi, Nucl. Instr. Meth. A 598, 59, 2009

[10] T. Kishishita, G. Sato, H. Ikeda, T. Takahashi, T. Kiyuna, Y. Mito, IEEE Trans. Nucl. Sci., 57, 2971, 2010

[11] G. Sato, T. Kishishita, H. Ikeda, T. Sakumura, T. Takahashi, IEEE Trans. Nucl. Sci, 58, 1370, 2011

[12] T. Takahashi, T. Mitani, Y. Kobayashi, M. Kouda, G. Sato, S. Watanabe, K. Nakazawa, Y. Okada, M. Funaki, R. Ohno, K. Mori, IEEE Trans. Nucl. Sci. NS-49, 1297, 2002 
[13] K. Nakazawa, T. Takahashi, S. Watanabe, G. Sato, M. Kouda, Y. Okada, T. Mitani, Y. Kobayashi, Y. Kuroda, M. Onishi, R. Ohno, H. Kitajima, Nucl. Instr. and Meth. A 512, 412, 2003

[14] H. Tajima, T. Nakamoto, T. Tanaka, S. Uno, T. Mitani, E. Silva, Y. Fukazawa, T. Kamae, G. Madejski, D. Marlow, K. Nakazawa, M. Nomachi, Y. Okada, T. Takahashi, IEEE Trans. Nucl. Sci. 51, 842, 2004 